\title{
A novel elastoplastic constitutive model for woven oxide/oxide ceramic matrix composites with anisotropic hardening
}

\author{
Hui Liu ${ }^{\mathrm{a}}$, Zhengmao Yang ${ }^{\mathrm{b}}$, Huang Yuan ${ }^{\mathrm{a}, *}$ \\ ${ }^{\text {a }}$ School of Aerospace Engineering, Tsinghua University, Beijing, China \\ ${ }^{\mathrm{b}}$ Institute of Mechanics, China Academy of Sciences, Beijing, China
}

\section{A R T I C L E I N F O}

\section{Keywords:}

Oxide/oxide ceramic matrix composite

Constitutive model

Plastic potential function

Anisotropic hardening

Asymmetric tension and compression

\begin{abstract}
A B S T R A C T
The oxide/oxide ceramic matrix composite exhibits superior mechanical properties in high temperature environment. However, complex nonlinear behavior is not clarified for high performance applications. The present work developed an elastoplastic constitutive model considering variations of hardening behavior over the stress states by introducing a novel loading function. The model can account for heterogeneous initial yield and complex hardening behavior accurately and was verified from experiments under tension and compression conditions with various off-axis angles. The computational results agree with stress-strain responses containing softening up to failure under arbitrary in-plane loading conditions.
\end{abstract}

\section{Introduction}

On account of the superior performance of high temperature stability, high specific strength and stiffness, the ceramic material is able to be used under extreme environmental conditions, but limited by its brittleness [1-4]. The ceramic matrix composites (CMC) improve the mechanical property by overcoming the inherent brittleness and found applications in important hot structures, such as wing leading edges of re-entry vehicles, nose caps etc. [5-7]. CMC will find increasing applications in aerospace propulsion components owing to the increasing need for lightweight structures. With further increasing operation temperature, the composite becomes more important for new generation of aero engines and is perhaps the sole material candidate for service temperature higher than $1200^{\circ} \mathrm{C}$ [8]. Oxide/oxide ceramic matrix composites exhibit lower maximum application temperature than $\mathrm{SiC} / \mathrm{SiC}$ composites, but are much easier for fabrication, less expensive and can additionally provide high resistance against oxidation and corrosion than other non-oxide ceramic matrix composites [9-11]. Considering the wide application prospects of the oxide/oxide ceramic matrix composite, it is imperative to study mechanical characterization of CMC.

Heterogeneity and quasi-brittleness are characteristic features of oxide/oxide CMC [12]. The material exhibits linear mechanical response when loading in the fiber directions, however inelastic deformations occur at a much lower loading level under off-axis tension or compression conditions $[13,14]$. The material behavior is extremely sensitive to the loading direction. Thermo-mechanical loads lead CMC to very different failure mechanisms and need detailed understanding of the mechanical behavior as well as damage evolution $[15,16]$. Great attentions have been paid to the complex nonlinear phenomena of CMC. The microstructure, such as fiber reinforcement, induces heterogeneous inelastic behavior, distinct deformations and failure mechanisms $[17,18]$. Additionally the material reveals significantly different compressive yield behavior from the tension state $[14,19,20]$. The complex material property makes conventional plasticity inefficient even inapplicable in analysis of the composite structures.

In the past decades, different constitutive models for CMC were proposed including micro/meso-scale models and macro-scale models. In the micro/meso-scale models, the properties for each composite component as well as interactions are considered, elastic properties [21], stress-strain response [22-24], damage process and failure mechanism [25-27] could be simulated based on these models. However it is difficult to determine the parameters in these models and to establish relationships between the macroscopic and microscopic mechanical behavior which limits the applications of micro/meso-scale models. Thus, the composites are considered to be homogeneous and the internal mechanisms of the nonlinear behaviors are out of consideration, macro-scale linear elastic models are still widely used to predict the mechanical response in general loading conditions due to the simplicity and usability.

As one of phenomenological constitutive models, anisotropic plasticity was widely used to describe the nonlinear behavior of composites

\footnotetext{
* Corresponding author.

E-mail address: yuan.huang@tsinghua.edu.cn (H. Yuan).
} 


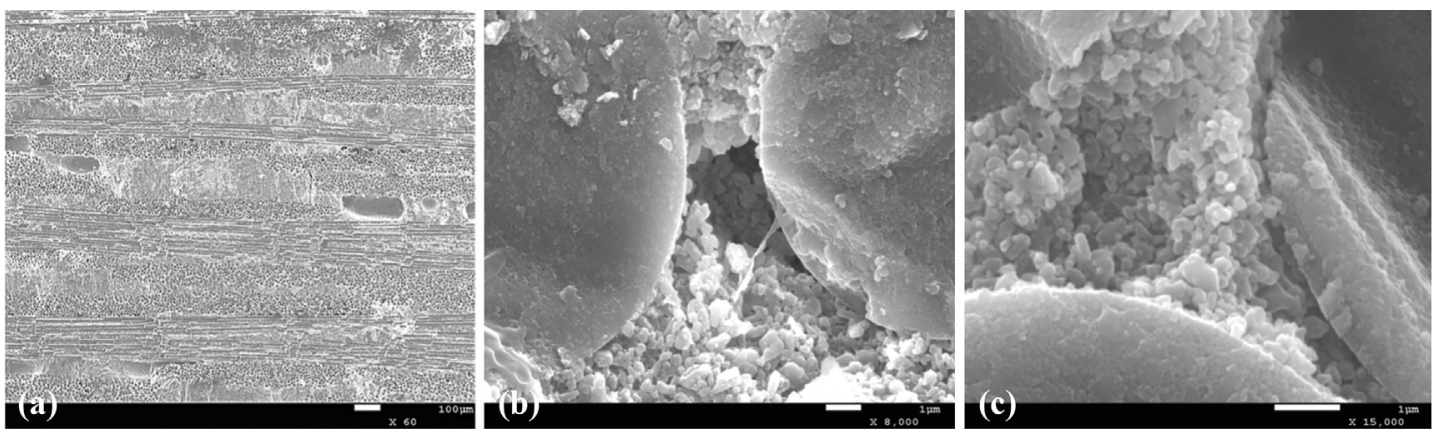

Fig. 1. Microstructure of the ceramic matrix composite. (a) The water jet cutting surface. Micro-voids are from manufacturing. (b) The ceramic fibers on the cutting surface. (c) The porous matrix consists of ceramic particles of $50 \mathrm{~nm}$. The matrix porosity reaches ca. $50 \mathrm{vol} \%$.

$[28,29]$ for monotonic loading conditions. The yield surface is essential to establish a plasticity constitutive model. The Hill-type yield criterion was adopted in these models. a one-parameter yield function was developed for unidirectional composite by Sun and Chen [28] which was valid for describing the nonlinear behaviors of unidirectional composite. This concept was extended to study woven composites in threedimensional conditions [29]. The nonlinear behaviors under tension conditions can be predicted accurately based on these models. However, fiber reinforced composites exhibit heterogeneous orientationdependent mechanical responses, including the initial yield behavior under tension and compression loading [18], the tension and compression asymmetry, which cannot be considered in these models accurately by the yield function in the quadratic form of the stress component.

In order to describe the asymmetric plastic behavior in tension and compression stress state, the hydrostatic pressure is introduced into the yield function [30,31]. Vyas et al. [32] proposed a three-dimensional plasticity-based constitutive model for unidirectional plies. The yield criterion is hydrostatically sensitive and can predict the mechanical behaviors in the fiber direction accurately. However it should be noted that the normal stress along fiber directions influences the shape of the yield surface in multi-axial loading conditions, which was neglected in Vyas' model. In plastic deformation of composites including the oxide/ oxide CMC, the strain softening occurs [33,34]. During the gradually softening process of the composite, the anisotropy of the material become more significant. The softening behaviors are rarely discussed in the composite mechanics community.

In conventional plasticity for metals, the plastic strain hardening is considered to be unique which indicates that the hardening rule of the material is independent of the loading configuration [35-37]. This assumption is trivial for homogeneous metals, but not accurate for composites. Introducing a suitable mapping function for the hardening rule under arbitrary loading conditions becomes the major research task in the composite plasticity. Ogi [38] found that the ratio of plastic strains in different material directions varies during the loading process and proposed a plastic model to explain the phenomenon. However the isotropic hardening hypothesis was still adopted in the Ogi's model [38,39]. In the Vyas' model [32], a kinematic hardening law was adopted in place of the isotropic hardening law by introducing the backstress into the yield function, nevertheless it is difficult to determine the evolution of the backstress for the oxide/oxide CMC from common uniaxial tests because of the brittleness of the material in the fiber direction. The kinematic hardening law can additionally not describe changes of the yield surface for various loading paths. Thus, a further study of the anisotropic hardening modeling of the oxide/oxide $\mathrm{CMC}$ is necessary to relate the heterogeneous material behavior to different loads.

In the present work, an elastoplastic constitutive model is introduced to consider unilateral effects and anisotropic hardening behaviors in a $2 \mathrm{D}$ woven oxide/oxide ceramic matrix composite. The yield surface is assumed to be independent of loading configuration and a loading-angel-dependent hardening rule is adopted to describe the orientation-dependent inelastic behavior of the CMC material. The new anisotropic plastic constitutive model can describe the overall stressstrain response, including hardening and softening depending on loading angles. Different off-axis tension/compression tests as well as by a center-holed tensile panel confirm reasonable accuracy of the model.

\section{Materials and experiments}

\subsection{Specimens}

In the present work a 2D woven oxide/oxide ceramic matrix composite plate is investigated. The plate consists of 20 sheets of Nextel ${ }^{\mathrm{TM}}$ 610 fiber fabrics, formed by the matrix of $\mathrm{Al}_{2} \mathrm{O}_{3}-\mathrm{SiO}_{2}-\mathrm{ZrO}_{2}$ [9]. The textile sheets are interlaced by two sets of the fiber ply at right angle, the longitudinal fiber ply (the warp) and the lateral fiber ply (the weft). A fabric sheet is usually woven on a loom, a device that holds the warp fiber plies in place while weft pies are woven through them. The microstructure of the material can be learnt from the water jet cutting surface in Fig. 1. The specimen surface after water-jet cutting has little visible damage, as shown in the figure. The surface reveals clear fracture ends of the fibers as well as the matrix particles, since the material was broken by an extremely high force. The material with a raw density of $2.9 \mathrm{~g} / \mathrm{cm}^{3}$ contains a total porosity of $25 \mathrm{vol} \%$ to $35 \mathrm{vol} \%$. The fiber volume content of the composite is $35 \mathrm{vol} \%$ to $45 \mathrm{vol} \%$. That means, the matrix is porous with more than $50 \%$ pores.

The maximum application temperature of the oxide/oxide ceramic matrix composite is about $1200^{\circ} \mathrm{C}$, a higher temperature can cause obvious mechanical performance degradations, as studied in [9]. Due to the strong bending deformations in weaving, the warp is generally more damaged than the weft. The macroscopic behavior of a sheet is orthogonal with a higher stiffness in the weft direction. The ceramic matrix composite plate is plied up by 20 sheets which are rotated $90^{\circ}$ each other, so that the plate possesses the similar stiffness in both $0^{\circ}$ and $90^{\circ}$ direction. In the present work, the longitudinal and lateral directions are defined as $x$ axis and $y$ axis, respectively. $0^{\circ}$ specimens are on the $x$ axis and $90^{\circ}$ specimens are on the $y$ axis.

The initial material plate is $2.8 \mathrm{~mm}$ thick. The dog-bone-shaped specimen was designed for tension tests and the square specimen was used in compression tests, as shown in Fig. 2. The compressive test was performed with help of a frock clamp to prevent buckling. Both uniaxial on-axis $\left(0^{\circ}, 90^{\circ}\right)$ and off-axis $\left(30^{\circ}, 45^{\circ}, 60^{\circ}\right)$ specimens were fabricated and tested under monotonic tension and compression loadings to study the in-plane mechanical behavior.

Tension and compression specimens with off-axis angles $0^{\circ}, 30^{\circ}, 45^{\circ}$, $60^{\circ}$ were tested under monotonic loading conditions, with at least two specimens for each loading condition, as shown in Fig. 3. As oxide/ oxide ceramic matrix composites are weak matrix composites because 


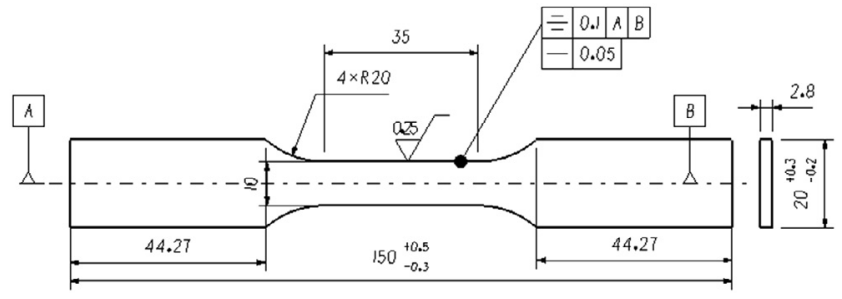

Fig. 2. Geometry of the uniaxial tension and compression specimens.

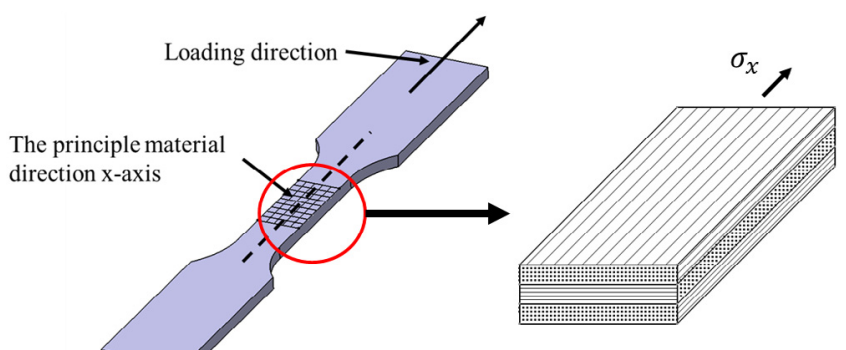

(a)

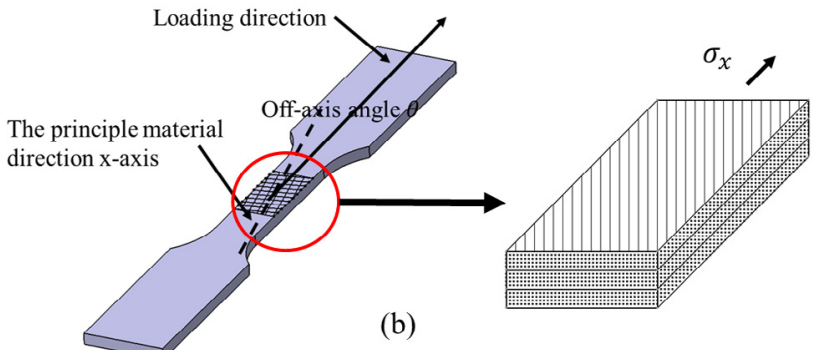

Fig. 3. The stress states of on-axis and off-axis specimens (a) on-axis monotonic tensile specimens (b) off-axis monotonic tensile specimens.

of the high porosity in the matrix, the tensile strength of the material is mainly dominated by fibers [24]. As fibers are laying along particular directions $\left(0^{\circ}\right.$ and $\left.90^{\circ}\right)$, the loading direction has great influences on the mechanical properties of the material.

Two strain gages were implemented to measure the strains along $x$ axis and $y$ axis, all the experiments are conducted on an MTS servohydraulic test machine under displacement control at room temperature. The loading speed of $0.001 \mathrm{~mm} / \mathrm{s}$ ensures a quasi-static loading process.

In the present work the shear modulus of the material was evaluated from a $45^{\circ}$ off-axis tensile test, in accordance with the ASTM standard D 3518 [40]. The axial strain $\varepsilon_{x}$ and the transverse strain $\varepsilon_{y}$ were measured by a strain gage rosette. According to [40], the shear modulus $G_{12}$ can be obtained from

$G_{12}=\frac{\sigma_{x}}{2\left(\varepsilon_{x}-\varepsilon_{y}\right)}$,

where $\sigma_{x}$ notes the tensile stress, and $\sigma_{x}=\frac{F}{A}$, where $A$ is the cross-sectional area in tensile direction of the specimen.

\subsection{Experimental results}

Based on the experimental tests, macroscopic mechanical behaviors of the woven ceramic matrix composite were studied under in-plane onaxis and off-axis tensile loading conditions. The stress-strain curves of monotonic tension for different off-axis angle specimens and the primary mechanical properties of the woven oxide/oxide ceramic matrix composite in uniaxial loadings were shown in Fig. 4 for both tension and compression. The characteristic values from the tests are, furthermore, summarized in Table 1, depending on the loading angle $\theta$. Obviously, the values varying with loading angle are not characteristic for the material and have to be correlated with a material representation.

In the figure the material does not show inelastic behavior in the onaxis direction $0^{\circ}$ and is essentially linearly elastic under both tension and compression till failure. The stress-strain relationship becomes severe elastic-plastic as soon as the loading is off-axis and the material behaves typical plastic hardening under tension. Before failure the material displays significant softening.

From the stress-strain response of different off-axis angle specimens shown in Fig. 4, it can be observed that the stress-strain curves for the off-axis specimens can be divided into three regimes before failure occurs: (1) The loading state is within the elastic region, that is, the stressstrain response of the material is nearly linear and the irreversible deformation is zero. (2) The stress state reaches the yield stress of the materials in this loading direction, that is, the stress-strain response comes into the second stage. In this stage, as the material is a weak matrix composite, micro cracks initiate and propagate in the matrix. Irreversible deformations begin to accumulate. The material demonstrates significant plastic deformations, depending on the loading direction. (3) In the third regime, the material is damaged by cracking in the matrix and delamination between the matrix and the fibre. The mechanical behavior shows softening and the stress decreases with increasing strain [13].

From the figure the mechanical behavior depends on the loading angle substantially. Whereas the fibre directions are elastic till fracture, the curve of $60^{\circ}$ shows large plastic deformations with very low yield stress. The material stiffness from tension tests varies with the loading angle and implies significant difference in elastic modulus of different directions. The different elastic moduli in $0^{\circ}$ and $90^{\circ}$ directions mean asymmetric micro-structures in the longitudinal and lateral plies. As the fibre plies in the $90^{\circ}$ direction are much more inflected than the fibers in $0^{\circ}$ direction because of the weaving technique, which leads to reduction of the effective fibre volume fraction in $90^{\circ}$ direction, the elastic moduli in the $90^{\circ}$ direction is small than that in the $0^{\circ}$ direction. The material becomes weaker in the off-axis direction and becomes typically elastic-plastic since the weak matrix takes part in resistance against applied loads.

Elastic modulus under compression is slightly smaller than that under tension which is related to the complex microstructure [13] and the material comes into yield at a higher stress level under compression. In Table 1, experimental results are summarized for different loading angles $\theta$ under both tension and compression.

\section{Constitutive model}

\subsection{Plasticity framework}

In this section, an elastoplastic constitutive model is proposed to describe the macroscopic nonlinear behavior of the woven oxide/oxide ceramic matrix composite, including asymmetric tension and compression behavior under multi-axial loading conditions. In the framework of elastic-plastic mechanics, the total strain increment can be decomposed into two parts, as

$\mathrm{d} \varepsilon=\mathrm{d} \varepsilon_{\mathrm{e}}+\mathrm{d} \varepsilon_{\mathrm{p}}$.

Above the plastic part $\mathrm{d} \varepsilon_{p}$ is discussed within the framework of plasticity. The elastic strain $\mathrm{d} \varepsilon_{e}$ is calculated based on elasticity theory of composites, as

$\mathrm{d} \varepsilon_{\mathrm{e}}=\mathbf{C}: \mathrm{d} \sigma$,

where $\sigma$ represents Cauchy stress tensor and $\mathbf{C}$ stands for the compliance matrix of the composite. For the orthotropic in-plane material, the equation can be written as 


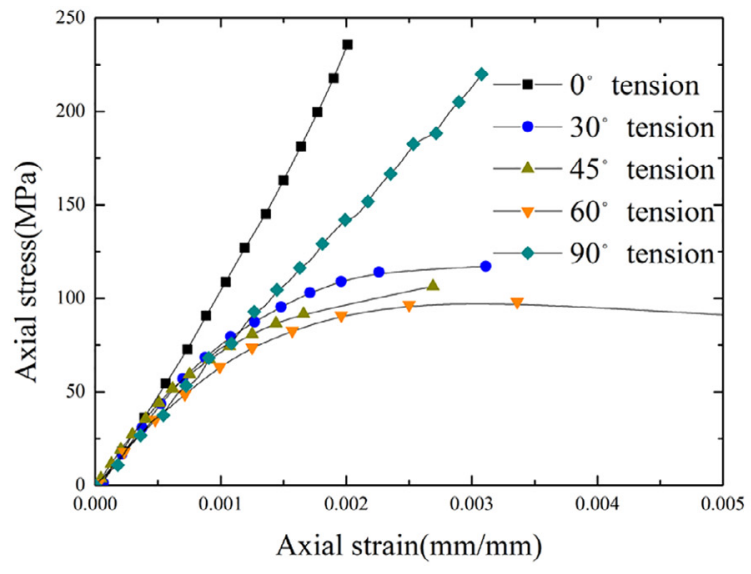

(a)

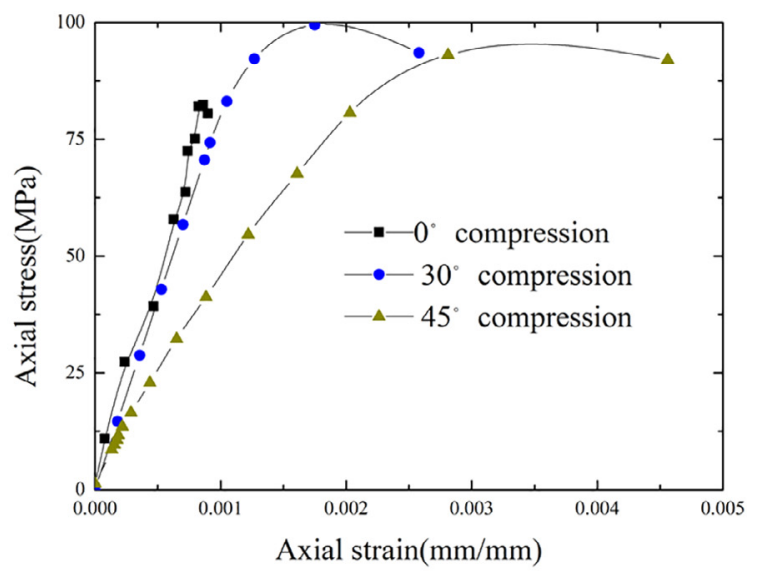

(b)

Fig. 4. Stress-strain curves depending on the off-axis loading angle. (a) Tension tests. (b) Compression tests.

Table 1

Mechanical properties of the oxide/oxide ceramic matrix composite in different directions.

\begin{tabular}{llllll}
\hline$\theta$ & $\begin{array}{l}\text { Elastic } \\
\text { modulus } \sigma_{\theta} \\
(\mathrm{GPa})\end{array}$ & $\begin{array}{l}\text { Yield } \\
\text { stress } \\
\sigma_{y \theta} \\
(\mathrm{MPa})\end{array}$ & $\begin{array}{l}\text { Fracture } \\
\text { stress } \sigma_{f \theta} \\
(\mathrm{MPa})\end{array}$ & $\begin{array}{l}\text { Failure } \\
\text { strain } \varepsilon_{f \theta} \\
(\%)\end{array}$ & $\begin{array}{l}\text { Ultimate } \\
\text { stress } \sigma_{u \theta} \\
(\mathrm{MPa})\end{array}$ \\
\hline $0^{\circ}$-tension & 120.43 & - & 254.26 & 0.21 & 254.26 \\
$30^{\circ}$-tension & 89.30 & 56.72 & 111.58 & 0.32 & 118.80 \\
$45^{\circ}$-tension & 75.41 & 48.67 & 103.43 & 0.44 & 110.54 \\
$60^{\circ}$-tension & 69.01 & 42.43 & 84.12 & 0.71 & 99.82 \\
$90^{\circ}$-tension & 72.35 & - & 220.51 & 0.31 & 220.51 \\
$0^{\circ}$-compression & 112.91 & - & 86.62 & 0.094 & 86.62 \\
$30^{\circ}$-compression & 89.01 & 79.47 & 95.82 & 0.25 & 100.61 \\
$45^{\circ}$-compression & 57.34 & 62.39 & 92.24 & 0.45 & 97.54 \\
\hline
\end{tabular}

$\left[\begin{array}{l}\mathrm{d} \varepsilon_{11}^{\mathrm{e}} \\ \mathrm{d} \varepsilon_{22}^{\mathrm{e}} \\ \mathrm{d} \varepsilon_{12}^{\mathrm{e}}\end{array}\right]=\left[\begin{array}{ccc}\frac{1}{E_{11}} & -\frac{\nu_{21}}{E_{11}} & 0 \\ -\frac{\nu_{21}}{E_{11}} & \frac{1}{E_{22}} & 0 \\ 0 & 0 & G_{12}\end{array}\right]\left[\begin{array}{l}\mathrm{d} \sigma_{11}^{\mathrm{e}} \\ \mathrm{d} \sigma_{22}^{\mathrm{e}} \\ \mathrm{d} \sigma_{12}^{\mathrm{e}}\end{array}\right]$.

Above $E_{11}$ and $E_{22}$ represent elastic modulus in the principal directions, i.e. the longitudinal $x$ and the lateral $y$-direction, respectively. $G_{12}$ represents the in-plane shear modulus, $\nu_{21}$ represents Poisson's ratio measured during the tension experiment in $x$ direction. Because of the asymmetry in the tension and compression material behavior, elastic modulus can be expressed as

$E_{11}= \begin{cases}E_{11}^{\mathrm{t}} & \sigma_{11}>0 \\ E_{11}^{\mathrm{c}} & \sigma_{11} \leqslant 0 .\end{cases}$

The elastic modulus in Direction $2 \mathrm{E}_{22}$ can be defined in the same way. Poisson ratio $\nu_{21}$ seems sensitive to the stress state. The material contraction under tension is significantly larger than that under compression, as shown in Table 2. It can be expressed as a function of the mean stress, as

$\nu_{21}= \begin{cases}\nu_{21}^{\mathrm{t}} & \sigma_{m}>0, \\ \nu_{21}^{\mathrm{c}} & \sigma_{m} \leqslant 0\end{cases}$

Table 2

Orthotropic moduli of the CMC.

\begin{tabular}{lllll}
\hline & $\mathrm{E}_{11}(\mathrm{GPa})$ & $\mathrm{E}_{22}(\mathrm{MPa})$ & $\nu_{21}$ & $\mathrm{G}_{12}(\mathrm{MPa})$ \\
\hline Tension & 120.04 & 67.16 & 0.05 & 32.59 \\
Compression & 112.79 & 56.72 & 0.02 & 32.87
\end{tabular}

where $\sigma_{m}=\sigma_{i i} / 3$. The stiffness matrix of the material is defined through $\mathbf{S}=\mathbf{C}^{-1}$. The values in Table 2 are representative for the material's elastic property in the principal directions and can be considered as the material constants for the orthogonal composite.

As the oxide/oxide matrix material is considered to be orthotropic, the orthotropic moduli of the material can be obtained from the linear segment of on-axis $\left(0 / 90^{\circ}\right)$ and off-axis $\left(30 / 45 / 60^{\circ}\right)$ tension curves. $E_{11}$ for tension and compression conditions is equal to the slope of the linear segment for the $0^{\circ}$ tension and compression tests listed in Table 1 and the poisson ratio is calculated from the longitudinal strain and transverse strain measured in the $0^{\circ}$ tests. The shear modulus $G_{12}$ is calculated from Eq. (1) according to the longitudinal strain and transverse strain measured in the $45^{\circ}$ tests. Furthermore, based on the elasticity theory, the relationship between the elastic modulus $E_{\theta}$ in the $\theta$ direction and the orthotropic moduli can be expressed as

$\frac{1}{E_{\theta}}=\frac{1}{E_{11}} \cos ^{4} \theta+\frac{1}{E_{22}} \sin ^{4} \theta-\left(\frac{1}{G_{12}}-\frac{2 \nu_{21}}{E_{11}}\right) \sin ^{2} \theta \cos ^{2} \theta$,

where $\theta$ means the off-axis angle. Should the off-axis modulus be measured, the orthotropic moduli can be calculated by solving the equation set.

The elastic stress-strain relationship is defined in the principal material coordinate system. A coordinate transformation matrix is defined to obtain the elastic stiffness matrix in the off-axis direction, which is defined as

$\mathbf{T}=\left[\begin{array}{lll}\cos ^{2} \theta & \sin ^{2} \theta & 2 \sin \theta \cos \theta \\ \sin ^{2} \theta & \cos ^{2} \theta & -2 \sin \theta \cos \theta \\ -\sin \theta \cos \theta & \sin \theta \cos \theta & \cos ^{2} \theta-\sin ^{2} \theta\end{array}\right]$,

where $\theta$ denotes the off-axis angle. The stiffness matrix under uniaxial loading conditions can be calculated from $\mathbf{S}_{\theta}=\mathbf{T}^{-1} \mathbf{S}\left(\mathbf{T}^{-1}\right)^{\mathrm{T}}$.

Sun and Chen [28] proposed a plastic potential function for unidirectional fiber reinforced composites, which is widely used in studying plastic behavior of composites, as

$f=\frac{1}{2} \sigma_{22}^{2}+a_{66} \sigma_{12}^{2}$

where the reinforced fibers are in the 1-coordinate. The yield function supposes there are no plastic strains in the fiber directions of composite, so that only one normal stress component affects yield behavior, which cannot describe the biaxial tension tests results of woven composites, as shown in Fig. 4.

In the present work the potential function in Eq. (9) is generalized to the woven composite with the warp and the weft principal directions, as 
$f=\sqrt{3\left[F\left(\sigma_{11}+\mu \sigma_{22}\right)^{2}+2 \sigma_{12}^{2}\right]}+k\left(\sigma_{11}+\sigma_{22}\right)$

with three model parameters, $F, \mu$ and $k$, related to the anisotropy of the composite and should be determined from experimental data. The stress components above are defined in the material principal coordinate system. The effective stress and the yield surface can be defined as

$f=\sigma_{y}$,

where yield stress $\sigma_{y}$ characterizes material resistance against plastic deformation and the hardening law to describe the evolution of subsequent yield stress.

For the associated plasticity the effective stress is assumed to satisfy $\bar{\sigma}=f$. The plastic strain can be obtained from

$\mathrm{d} \varepsilon_{i j}^{\mathrm{p}}=\mathrm{d} \lambda \frac{\partial f}{\partial \sigma_{i j}}$,

in which $\mathrm{d} \lambda$ is a scalar proportionality coefficient which determines the increment of the plastic flow.

\subsection{Plastic hardening}

According to the plasticity theory, the increment of the plastic work density is expressed as

$\mathrm{d} W_{\mathrm{p}}=\bar{\sigma} \mathrm{d} \bar{\varepsilon}_{\mathrm{p}}=\sigma_{i j} \mathrm{~d} \varepsilon_{i j}^{\mathrm{p}}$.

Above $\bar{\sigma}$ is the effective stress calculated by the yield function, $\mathrm{d} \bar{\varepsilon}_{\mathrm{p}}$ is the increment of the equivalent plastic strain, $\sigma_{i j}$ is the component of the stress tensor and $\mathrm{d} \varepsilon_{i j}^{\mathrm{p}}$ is the increment of the plastic strain tensor. The Einstein's summation convention is used in the present work.

Substituting the flow rule, Eq. (12), into Eq. (13) follows

$\mathrm{d} \bar{\varepsilon}_{\mathrm{p}}=\mathrm{d} \lambda$.

The equivalent plastic strain increment is equal to the proportionality factor in the flow rule. To establish the constitutive description of the composite, the stress-strain relation has to be correlated with tension and compression tests, which define relation between the effective stress and equivalent plastic strain,

$\sigma_{y}=\kappa\left(\bar{\varepsilon}^{\mathrm{p}}\right)$

with $\bar{\varepsilon}_{p}$ as the equivalent plastic strain, which is a material state variable and records the loading history. The correlation between the plastic strain and yield depends on loading direction. The power law is widely used to describe the hardening behavior of the composite materials and cannot not describe the softening. Hence the hardening function can be re-written as

$\kappa\left(\bar{\varepsilon}_{\mathrm{p}}\right)=A\left(\bar{\varepsilon}_{\mathrm{p}}\right)^{n} \exp \left(-m\left\langle\bar{\varepsilon}_{\mathrm{p}}-\varepsilon_{\mathrm{p}}^{0}\right\rangle^{t}\right)+\sigma^{0}$

where $\langle\cdot\rangle$ denotes the MacAuley brackets defined as $\langle x\rangle=(x+|x|) / 2$. Above $\sigma^{0}$ describes initial yield stress of the material and is assumed to be independent of the loading configuration. The orientation-dependence of the initial yielding is expressed in the potential function, Eq. (10). $\varepsilon_{P}^{0}$ characterizes softening in the stress-strain relationship. $A$ and $m$ stand for effects of loading orientation in plastic strain hardening. $n$ and $t$ are constants to be determined from experimental data.

In most published constitutive models the relationship between the effective stress and equivalent plastic strain is assumed to be independent of the loading configuration, that is, the hardening function is a function of equivalent plastic strain only [28,30,31]. Effects of the off-axis loads are considered in the definition of the effective stress, Eq. (10). It results in the experimental curves cannot directly be related in the computational model. In the present work, the off-axis experimental stress-strain curves are separated into initial yielding and plastic strain hardening. The initial yield stress variation is considered in the effective stress, whereas the plastic hardening is directly coupled with experimental stress-strain curves to obtained more realistic approaches. That is, the plastic hardening depends on both loading configuration and plastic strain, which can be characterized by a dimensionless variable, $\omega=\frac{\left|\sigma_{12}\right|}{\bar{\sigma}}$.

In the formulation the plastic hardening effects are expressed explicitly in Eq. (16). Influences of the off-axis loads have to be integrated into other parameters in Eq. (16) as functions of $\omega$.

\subsection{Representation of the off-axis loads}

The experimental data of the off-axis tension and compression tests are used to establish the representation of the off-axis behavior of the material. According to the stress transformation, the stresses in the principal material direction in uniaxial tension can be written as

$\sigma_{11}=\sigma_{\theta} \cos ^{2} \theta$,

$\sigma_{22}=\sigma_{\theta} \sin ^{2} \theta$

$\sigma_{12}=-\sigma_{\theta} \cos \theta \sin \theta$

where $\sigma_{\theta}$ denotes the off-axis tensile stress. Substituting the stress transformation into the effective stress $\bar{\sigma}$, follows

$\bar{\sigma}=\sigma_{\theta} h(\theta)$

where $h(\theta)$ describes effects of the off-axis uniaxial tension, as

$h(\theta)=$

$\operatorname{sign}\left(\sigma_{\theta}\right) \sqrt{3 F\left(\cos ^{2} \theta+\mu \sin ^{2} \theta\right)^{2}+6 \sin ^{2} \theta \cos ^{2} \theta}+k$.

The equation above establishes relation between the effective stress and the tensile stress in an off-axis tension test. In analogy to the derivation of the stress representations, the strain transformation can be written as

$\mathrm{d} \varepsilon_{\theta}^{\mathrm{p}}=\mathrm{d} \varepsilon_{11}^{\mathrm{p}} \cos ^{2} \theta+\mathrm{d} \varepsilon_{22}^{\mathrm{p}} \sin ^{2} \theta-\mathrm{d} \varepsilon_{12}^{\mathrm{p}} \cos \theta \sin \theta$.

Substituting Eq. (21) into the equivalence of the plastic work, Eq. (13), the equivalent plastic strain increment can be expressed as

$\mathrm{d} \bar{\varepsilon}_{\mathrm{p}}=\mathrm{d} \varepsilon_{\theta}^{\mathrm{p}} / h(\theta)$.

Under the proportional loading condition, the expression of the equivalent plastic strain can further be written as

$\bar{\varepsilon}=\varepsilon_{\theta}^{\mathrm{p}} / h(\theta)$.

From an off-axis tensile test the stress measured, $\sigma_{\theta}$, is expressed as a function of the off-axis strain $\varepsilon_{\theta}^{\mathrm{p}}$, as

$\sigma_{\theta}=\kappa_{\theta}\left(\varepsilon_{\theta}^{\mathrm{p}}\right)$,

where $\kappa_{\theta}$ is the hardening function for the present off-axis angle. Combining the results with Eq. (26) follows

$$
\begin{aligned}
\bar{\sigma} & =\kappa_{\theta}\left(\varepsilon_{\theta}^{\mathrm{p}}\right) h(\theta) \\
& =\kappa_{\theta}\left(\bar{\varepsilon}_{\mathrm{p}} h(\theta)\right) h(\theta) .
\end{aligned}
$$

Equation above denotes the formal stress-strain relationship, depending on the off-axis angle. Due to the heterogeneity of the material behavior, the effective stress is not uniquely determined by the equivalent plastic strain alone, but the explicit dependence on the off-axis loading angle cannot directly be derived.

In the present work, different tension tests with the off-axis angle $\theta$ equal to $0^{\circ}, 45^{\circ}, 60^{\circ}$ and $90^{\circ}$ as well as compression tests with the offaxis angle $\theta$ equal to $0^{\circ}, 30^{\circ}, 45^{\circ}$ were performed to determine the plastic hardening function $\kappa_{\theta}$, depending on the off-axis angle $\theta$. Furthermore, the hardening function $\kappa\left(\bar{\varepsilon}_{\mathrm{p}}\right)$ can be identified. In analogy to $\kappa\left(\bar{\varepsilon}_{\mathrm{p}}\right)$, the off-axis stress-strain curves can be approximated by the expression as

$\sigma_{\theta}=A_{\theta}\left(\varepsilon_{\theta}^{\mathrm{p}}\right)^{n_{\theta}} \exp \left(-m_{\theta}\left\langle\varepsilon_{\theta}^{\mathrm{p}}-\varepsilon_{\theta}^{0}\right\rangle^{t_{\theta}}\right)+\sigma_{\theta}^{0}$,

where $A_{\theta}, n_{\theta}, t_{\theta}, \varepsilon_{\theta}^{0}, m_{\theta}$ and $\sigma_{\theta}^{0}$ are the fitting parameters related to the off-axis angle and can be determined from experimental data. The index 


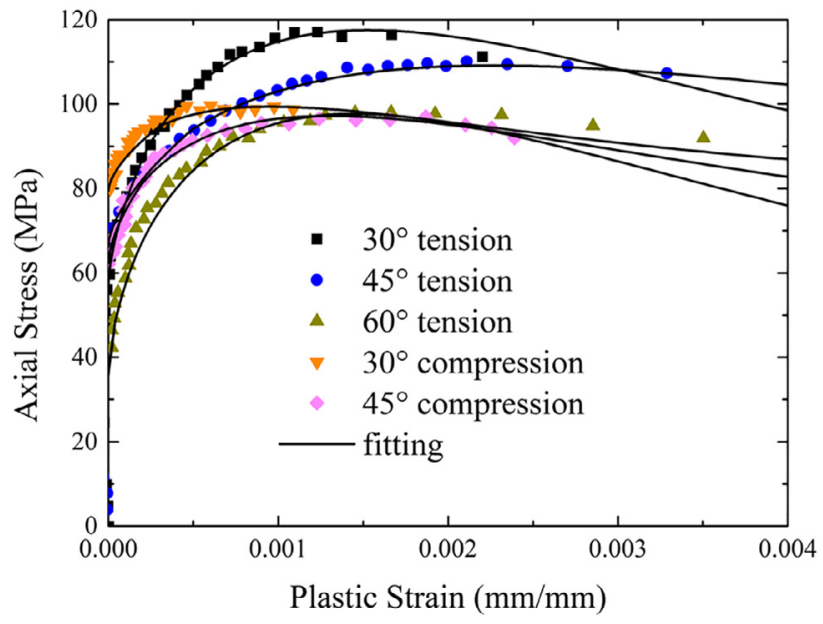

Fig. 5. The axial stress-plastic strain curves under different off-axis angles.

$\theta$ represents the dependence on the off-axis angle. Comparing with Eq. (16), the model parameters of the expression $\kappa\left(\bar{\varepsilon}^{p}\right)$ can be related to fitting parameters, as

$$
\begin{aligned}
A(\theta) & =A_{\theta}(\theta)[h(\theta)]^{n+1}, \\
m(\theta) & =m_{\theta}(\theta) h(\theta), \\
\varepsilon^{0} & =\varepsilon_{\theta}^{0}(\theta) / h(\theta), \\
\sigma^{0} & =\sigma_{\theta}^{0}(\theta) h(\theta) .
\end{aligned}
$$

The expressions above suggest that $n_{\theta}$ and $t_{\theta}$ are independent of $\theta$. Fig. 5 shows $\sigma_{\theta}-\varepsilon_{\theta}^{\mathrm{p}}$ curves together with the fitting results of Eq. (28) for different off-axis loads. The identified fitting parameters are summarized in Table 3 , in which $n_{\theta}=0.62$ and $t_{\theta}=0.95$. The initial yield stress $\sigma_{\theta}^{0}$ depends on the loading angle and has to meet the constant yield stress $\sigma^{0}=74.77 \mathrm{MPa}$.

Variations of the initial yield stress $\sigma_{\theta}^{0}$ are shown in Fig. 6 in a polar coordinate system. From the figure the yield stress reaches its maximum in ca. $40^{\circ}$. The symmetry of the yield surface about $90^{\circ}$ is reasonable for the orthotropic composite. The asymmetric tension-compression explains the difference in material deformations, which is induced by the sign function in Eq. (22).

The initial yield surface in Eq. (10) is similar to the 3D DruckerPrager model, the hydrostatic pressure influences on the yield behavior of the ceramic matrix composite. The asymmetry of the yield surface means that $\sigma_{11}$ and $\sigma_{22}$ have different influences on the material plastic behavior. The yield surface also indicates that the shear stress $\sigma_{12}$ may possess great influences on plastic deformation of the material. The parameters $F, \mu$ and $k$ in the yield function can be determined, which are independent of $\theta$, as $F=0.6144, \mu=-1.88172$ and $k=0.18562$. Based on formulations above the orientation dependence of the stressstrain tension can be considered accurately, as shown in Fig. 5. However, in the constitutive model effects of the off-axis angle in the $\sigma_{\theta}-\varepsilon_{\theta}$ curves must be expressed by a loading state variable, such as $\omega$.

According to Fig. 6, the initial yield stresses at $0^{\circ}$ and $90^{\circ}$ is not infinite, that is, the material can come into plastic at $0^{\circ}$ and $90^{\circ}$, but with so strong hardening, that the whole stress-strain curve remains

Table 3

Fitting parameters for different off-axis loads.

\begin{tabular}{lllll}
\hline & $A_{\theta}$ & $m_{\theta}$ & $\varepsilon_{\theta}^{0}$ & $\sigma_{\theta}^{0}$ \\
\hline $30^{\circ}$-Tension & 5876.51 & 306.70 & 0.00023 & 56.72 \\
$45^{\circ}$-Tension & 3104.33 & 209.25 & 0.0005 & 48.67 \\
$60^{\circ}$-Tension & 7946.78 & 414.64 & 0.00015 & 42.43 \\
$30^{\circ}$-Compression & 2323.17 & 473.59 & 0.00025 & 79.47 \\
$45^{\circ}$-Compression & 3712.11 & 355.23 & 0.00045 & 62.39 \\
\hline
\end{tabular}

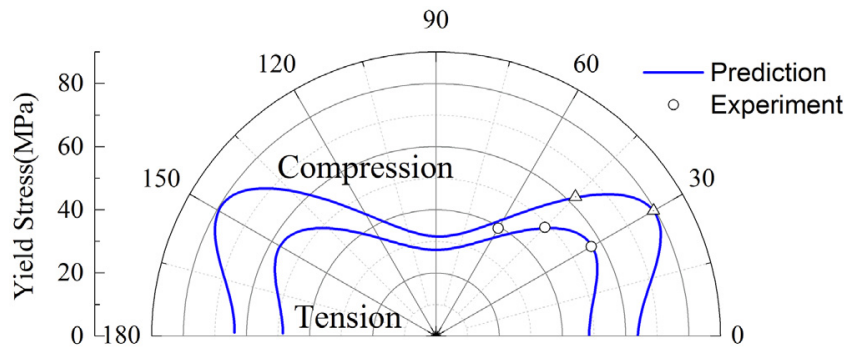

Fig. 6. The initial yield stress $\sigma_{\theta}^{0}$ as a function the off-axis angles.

linear. Such behavior is modeled in the constitutive model by $\omega=\left|\sigma_{12}\right| / \bar{\sigma}$. For both on-axis loads $\omega=0, A$ and $m$ in the hardening law go to infinity, which means that a tiny plastic strain increment causes dramatic stress increments. Such postulate makes computations stable and needs further experimental verifications.

Based numerical experiments, a quadratic function of $1 / \omega$ is introduced to describe $m$ and a linear function of $1 / \omega$ is used for $A$, as

$A=\frac{A_{1}}{\omega}+A_{0}, \quad m=\left(\frac{m_{1}}{\omega}+m_{2}\right)^{2}+m_{0}$.

The interpolations are plotted in Fig. 7, together with the experimental data.

The constitutive model has been implemented into the commercial finite element code ABAQUS via the user defined material interface UMAT. The present constitutive model based on the modified potential function, Eq. (10), and the off-axis dependence is expressed in the hardening rule, so that the radial return mapping algorithm can be applied to finish the stress integration using the backward Euler method. The correction of the off-axis angle is performed after integrating the increment step by updating $\omega$.

\section{Computational verifications}

The constitutive model developed in the previous section is implemented into the commercial finite element code ABAQUS and used to compute the stress-strain curves of different off-axis specimens. Fig. 8 summarizes results of uniaxial tension and compression tests with various off-axis angles $0^{\circ} / 15^{\circ} / 30^{\circ} / 60^{\circ} / 75^{\circ} / 90^{\circ}$. Both axial strain $\varepsilon_{x}$ and transverse strain $\varepsilon_{y}$ are plotted in the figure. Symbols represent the test data and curves are finite element computations. Additional computational results with $15^{\circ}$ and $75^{\circ}$ are provided although there are no corresponding experiments available. The development of the stressstrain curves seems to be reasonable.

The figures show that the nonlinearity of stress-strain decreases with loading direction approaches fibre directions and the softening in the stress-strain curve appears earlier, as demonstrated in the curves of

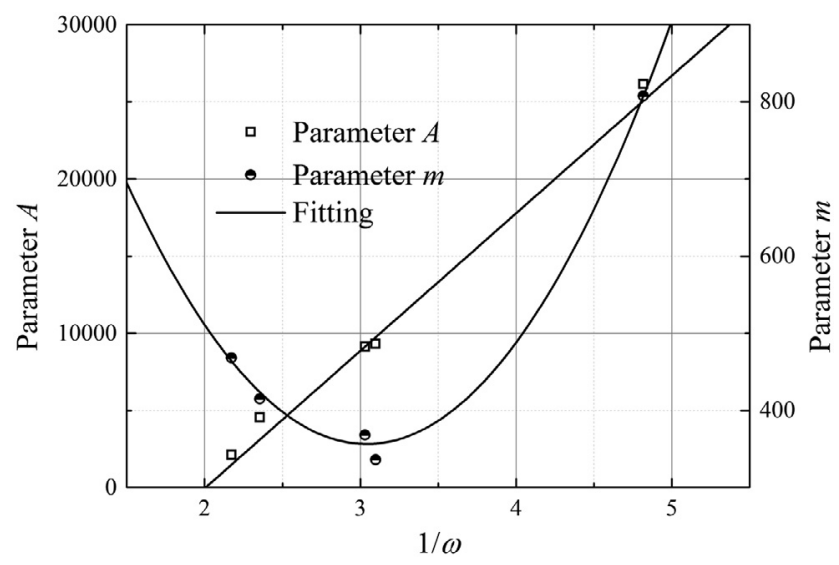

Fig. 7. The parameters $A$ and $m$ as functions of the off-axis variable $\omega$. 


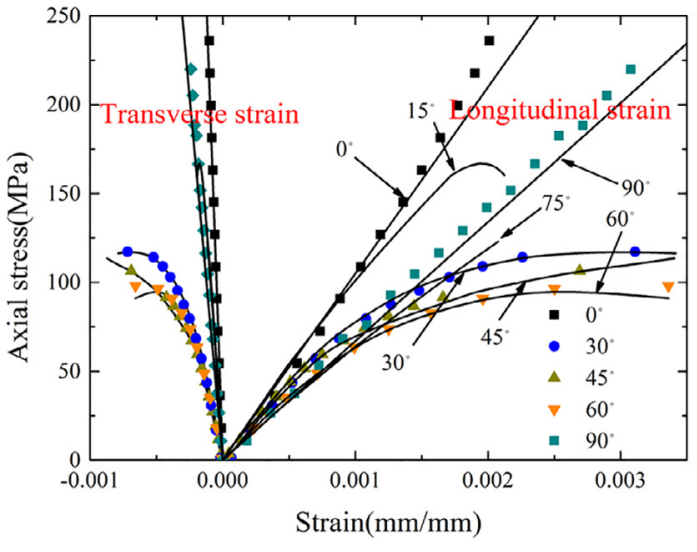

(a) Tension

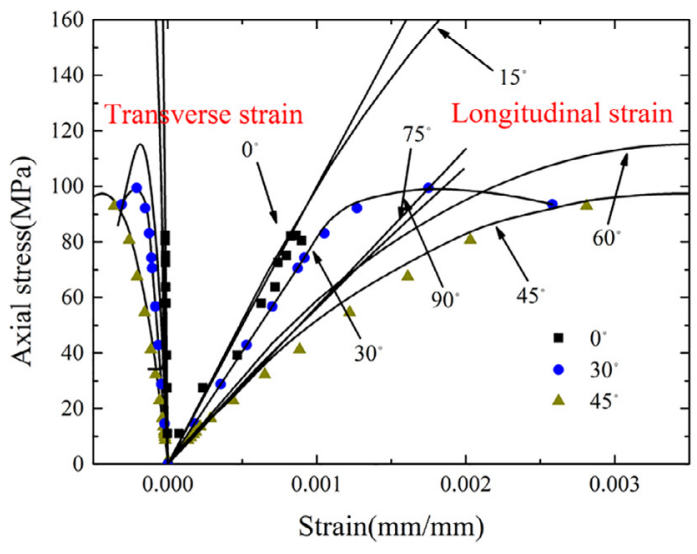

(b) Compression

Fig. 8. Predictions of the uniaxial tension and compression behavior of the CMC material.

$15^{\circ}$ and $75^{\circ}$. The stress drops rapidly when the tensile/compression strength is reached. It implies the brittle behavior of the material in the fiber direction. The shear stress induces plastic deformations and reduces loading capacity into the material. The difference between $0^{\circ}$ and $90^{\circ}$ is related to difference of the warp from the lateral fiber woven microstructure in the material. All experiments agree with computations reasonably. Computations give a reasonable prediction of the heterogeneous mechanical behavior of the material.

To further verify the accuracy of the proposed constitutive model, a holed specimen was tested under tensile loading to validate the proposed model. The experiment was conducted under displacement control with a velocity of $0.001 \mathrm{~mm} / \mathrm{s}$. Strains near the hole were measured by a strain rosette, as shown in Fig. 9 .

The tensile test was simulated by using the constitutive model developed in the previous section. The load vs. displacement curve in Fig. 10 confirms agreement between the constitutive model prediction and test results for the whole loading process. The softening load is caught accurately which was predicted from the uniaxial tension. Computation reveals that the maximum stress and strain around the hole appear in the direction between $30^{\circ}$ and $45^{\circ}$.

More computational results are plotted in Fig. 10, in which the development of the strains in a critical position of the specimen are illustrated. The strains grow with applied tensile displacement linearly at beginning, since the material behaves elastically. As soon as the material starts to be plastified, the shear strain increases more rapidly than the normal strain, which implies that the material's plastification is driven by the shear stress. As the applied load approaches the maximum load, the material of the critical place begins to softening induced by microstructural damage. In this stage, the strains increase overproportionally.

\section{Conclusions}

The oxide/oxide ceramic matrix composite possesses a novel high temperature property. From experiments the material displayed

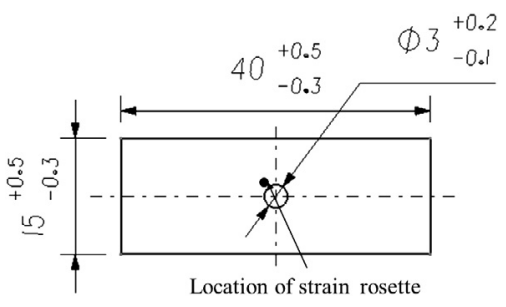

Fig. 9. Schematic of open-hole specimen.

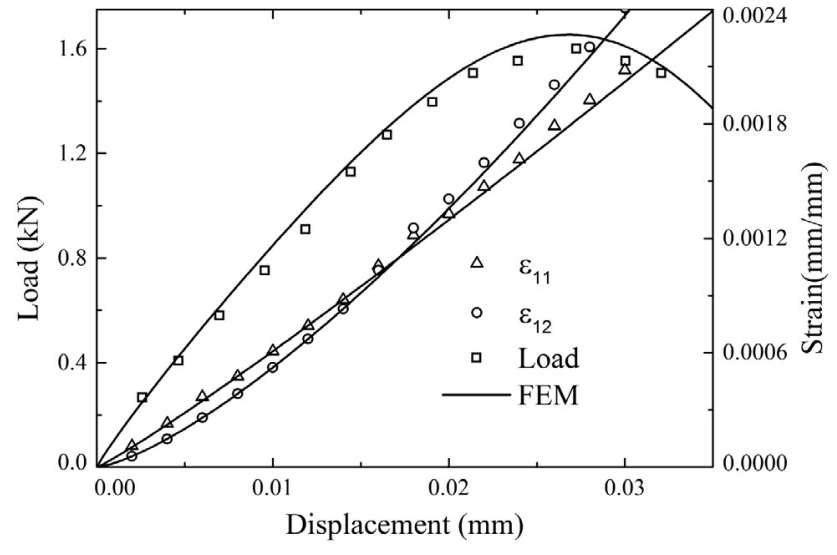

Fig. 10. Comparison of FEM calculation and experimental results.

significant inelastic behavior related to plastic deformations as well as material degradation. Under monotonic loading conditions, however, the inelastic material property can be described by the plasticity uniformly, without introducing the damage as additional variables. In the present work an anisotropic elastic-plastic constitutive model is developed and verified based on extensive on-axis and off-axis experimental results for a woven ceramic matrix composite. The following conclusions can be drawn:

- The constitutive equation developed in the present work decomposes the off-axis effects into initial yield and plastic hardening. Whereas the angle dependence of the initial yield condition is considered in the effective stress, the plastic hardening is directly taken into account in formulation of the hardening rule. The latter is related to the off-axis tensile test, which provides the possibility to compare the hardening equation with experimental records directly.

- Effects of the off-axis loads are represented by the normalized shear stress, as in the $\bar{\sigma}$-d $\bar{\varepsilon}_{\mathrm{p}}$ diagram. In the present two-dimensional cases, such formulation provides a simple correlation between the off-axis load and the material's principal direction. The model generates accurate results.

- The flexible formulation of the hardening rule allows to consider strain softening as well as other more complex mechanical behavior during plastic loading. The experimental verification confirms efficient convergence and high accuracy in computations.

- Introduction of the orientation-dependent hardening rule into the constitutive model provides a direct strategy to construct anisotropic plasticity for composites. However, this strategy is perhaps 
only efficient for 2D materials or thin plates and needs further verifications based on more extensive experimental and computational investigations.

\section{Acknowledgement}

The present work is financed by the China Natural Science Foundation under the contract numbers 11572169 and 51775294.

\section{Appendix A. Supplementary data}

Supplementary data associated with this article can be found, in the online version, athttps://doi.org/10.1016/j.compstruct.2019.111420.

\section{References}

[1] Marshall DB, Cox BN. Integral textile ceramic structures. Annu Rev Mater Res 2008;38:425-43.

[2] Pichon T, Barreteau R. Thermal protection systems: Heritage-development statusperspectives. 18th AIAA/3AF International Space Planes and Hypersonic Systems and Technologies Conference 2012:5846.

[3] Tang S, Hu C. Design, preparation and properties of carbon fiber reinforced ultrahigh temperature ceramic composites for aerospace applications: a review. J Mater Sci Technol 2017;33:117-30.

[4] Knoche R, Werth E, Weth M, García JG, Wilhelmi C, Gerendás M. Design and development approach for gas turbine combustion chambers made of oxide ceramic matrix composites, Mechanical Properties and Performance of Engineering Ceramics and Composites VI: Ceramic Engineering and Science Proceedings 32

[5] Ferraiuolo M, Scigliano R, Riccio A, Bottone E, Rennella M. Thermo-structural design of a ceramic matrix composite wing leading edge for a re-entry vehicle. Compos Struct 2019;207:264-72.

[6] Borrelli R, Riccio A, Tescione D, Gardi R, Marino G. Thermo-structural behaviour of an UHTC made nose cap of a reentry vehicle. Acta Astronaut 2009;65(3):442-56.

[7] Bansal NP, Lamon J. Ceramic matrix composites: materials, modeling and technology. John Wiley \& Sons; 2014.

[8] Njuguna J. Lightweight composite structures in transport: design, manufacturing, analysis and performance. Elsevier; 2016.

[9] Volkmann E, Tushtev K, Koch D, Wilhelmi C, Göring J, Rezwan K. Assessment of three oxide/oxide ceramic matrix composites: mechanical performance and effects of heat treatments. Compos Part A: Appl Sci Manuf 2015;68:19-28.

[10] Marshall D, Davis J. Ceramics for future power generation technology: fiber reinforced oxide composites. Curr Opin Solid State Mater Sci 2001;5:283-9.

[11] Blugan G, Strehler C, Vetterli M, Ehrle B, Duttlinger R, Blösch P, Kuebler J. Performance of lightweight coated oxide ceramic composites for industrial high speed wood cutting tools: a step closer to market. Ceram Int 2017;43:8735-42.

[12] Bansal NP. Handbook of ceramic composites. Springer Science \& Business Media; 2006. Vol. 200.

[13] Koch D, Tushtev K, Grathwohl G. Ceramic fiber composites: experimental analysis and modeling of mechanical properties. Compos Sci Technol 2008;68:1165-72. recent Advances in Experimental and Applied Research of Composite Materials.

[14] Ramdane CB, Julian-Jankowiak A, Valle R, Renollet Y, Parlier M, Martin E, Diss P. Microstructure and mechanical behaviour of a nextelTM610/alumina weak matrix composite subjected to tensile and compressive loadings. J Eur Ceram Soc 2017;37:2919-32.

[15] Yang Z, Yuan H, Liu H. Evolution and characterization of cyclic thermal shockinduced thermomechanical damage in oxide/oxide ceramics matrix composites. Int J Fatigue 2019;120:150-61.

[16] Yang Z, Yuan H, Markert B. Representation of micro-structural evolution and thermo-mechanical damage in thermal shocked 2-D woven oxide/oxide ceramic matrix composites. Int J Fatigue 2019;126:122-9.

[17] Baste S. Inelastic behaviour of ceramic-matrix composites. Compos Sci Technol 2001;61:2285-97.

[18] Bishara M, Rolfes R, Allix O. Revealing complex aspects of compressive failure of polymer composites - part i: fiber kinking at microscale. Compos Struct 2017;169:105-15.

[19] Wang M, Laird C. Damage and fracture of a cross woven C/SiC composite subject to compression loading. J Mater Sci 1996;31:2065-9.

[20] Hoppel CPR, Bogetti TA, John J, Gillespie W. The effect of tension compression asymmetry on modelling the bending response of sheet moulding compound composites. Compos Part B: Eng 2018;154:157-65.

[21] Wen PH, Aliabadi MH. Analytical model application for prediction of mechanical properties of natural fiber reinforced composites. Mater Today: Proc 2018;5:5809-18.

[22] Zhang D, Hayhurst D. Stress-strain and fracture behaviour of 0/90 and plain weave ceramic matrix composites from tow multi-axial properties. Int J Solids Struct 2010;47:2958-69.

[23] Choi H, Heinrich C, Ji W. An efficient homogenization technique for fiber tows in textile composites with emphasis on directionally dependent nonlinear stress-strain behavior. Compos Struct 2019;208:816-25.

[24] He T, Liu L, Makeev A, Shonkwiler B. Characterization of stress-strain behavior of composites using digital image correlation and finite element analysis. Compos Struct 2016;140:84-93.

[25] Tabiei A, Jiang Y. Woven fabric composite material model with material nonlinearity for nonlinear finite element simulation. Int J Solids Struct 1999;36:2757-71.

[26] Ye J, Qiu Y, Chen X, Ma J. Initial and final failure strength analysis of composites based on a micromechanical method. Compos Struct 2015;125:328-35.

[27] Li Z, Ghosh S, Getinet N, O’Brien DJ. Micromechanical modeling and characterization of damage evolution in glass fiber epoxy matrix composites. Mech Mater 2016;99:37-52.

[28] Sun C, Chen J. A simple flow rule for characterizing nonlinear behavior of fiber composites. J Compos Mater 1989;23:1009-20.

[29] Xie M, Adams DF. A plasticity model for unidirectional composite materials and its applications in modeling composites testing. Compos Sci Technol 1995;54:11-21.

[30] Cho J, Fenner J, Werner B, Daniel I. A constitutive model for fiber-reinforced polymer composites. J Compos Mater 2010;44:3133-50.

[31] Mandel U, Taubert R, Hinterhölzl R. Three-dimensional nonlinear constitutive model for composites. Compos Struct 2016;142:78-86.

[32] Vyas G, Pinho S, Robinson P. Constitutive modelling of fibre-reinforced composites with unidirectional plies using a plasticity-based approach. Compos Sci Technol 2011;71:1068-74.

[33] Flatscher T, Pettermann H. A constitutive model for fiber-reinforced polymer plies accounting for plasticity and brittle damage including softening - implementation for implicit FEM. Compos Struct 2011;93:2241-9.

[34] Ge Ingran, He C, Liang J, Chen Y, Fang D. A coupled elastic-plastic damage model for the mechanical behavior of three-dimensional (3d) braided composites. Compos Sci Technol 2018;157:86-98.

[35] Sun J, Yuan H. Cyclic plasticity modeling of nickel-based superalloy Inconel 718 under multi-axial thermo-mechanical fatigue loading conditions. Int J Fatigue 2019;119:89-101.

[36] Ma S, Yuan H. A continuum damage model for multi-axial low cycle fatigue of porous sintered metals base $\mathrm{d}$ on the critical plane concept. Mech Mater 2017;104:13-25.

[37] Yuan H, Zhang L, Ma S. Damage evolution and characterization for sintered powder metals with the varying porosity. Eng Fract Mech 2019;207:86-98.

[38] Ogi K, Takeda N. Effects of moisture content on nonlinear deformation behavior of CF/epoxy composites. J Compos Mater 1997;31:530-51.

[39] Xie J, Fang G, Chen Z, Liang J. An anisotropic elastoplastic damage constitutive model for 3d needled c/c-sic composites. Compos Struct 2017;176:164-77.

[40] ASTM-D3518. Standard test method for in-plane shear response of polymer matrix composite materials by tensile test of a \pm 45 laminate, American Society for Testing and Materials D3518/D3518M. 